\title{
A COMPACT SYMMETRIC SYMPLECTIC NON-KAEHLER MANIFOLD
}

\author{
EUGENE LERMAN
}

\begin{abstract}
In this paper I construct, using off the shelf components, a compact symplectic manifold with a non-trivial Hamiltonian circle action that admits no Kaehler structure. The non-triviality of the action is guaranteed by the existence of an isolated fixed point.

The motivation for this work comes from the program of classification of Hamiltonian group actions. The Audin-Ahara-Hattori-Karshon classification of Hamiltonian circle actions on compact symplectic 4-manifolds showed that all of such manifolds are Kaehler. Delzant's classification of $2 n$-dimensional symplectic manifolds with Hamiltonian action of $n$ dimensional tori showed that all such manifolds are projective toric varieties, hence Kaehler. An example in this paper shows that not all compact symplectic manifolds that admit non-trivial Hamiltonian torus actions are Kaehler. Similar techniques allow us to construct a compact symplectic manifold with a Hamiltonian circle action that admits no invariant complex structures, no invariant polarizations, etc.
\end{abstract}

\section{Introduction}

The main goal of this paper is to construct a nontrivial example of a Hamiltonian group action on a compact symplectic manifold which is not Kaehler.

The motivation for this work comes from the program of classification of Hamiltonian group actions. Delzant proved that if a compact symplectic manifold admits a completely integrable torus action then the manifold is a projective toric variety and so, in particular, it is Kaehler. Karshon has recently finished a classification of Hamiltonian circle actions on compact symplectic 4-manifolds [Ka], the classification that was initiated by Audin and by Ahara and Hattori. All such 4-manifolds turn out to be Kaehler. This should be contrasted with Gompf's results [Gom]. One naturally wonders to what extent symmetries force symplectic manifolds to be Kaehler.

Ideally a nontrivial example of a non-Kaehler circle symmetry would be an example of a Hamiltonian action all of whose fixed points are isolated. This would rule out such examples as products of compact non-Kaehler

Received February 14, 1996.

The author was partially supported by an NSF postdoctoral fellowship. 
symplectic manifolds with a two-sphere rotated about a fixed axis. Since I started the work on this project, there have been two interesting developments. S. Tolman constructed a compact symplectic 6 manifold which admits a Hamiltonian action of a 2-torus with isolated fixed points and which doesn't admit any invariant Kaehler structure [To]. C. Woodward showed that Tolman's manifold admits a Hamiltonian action of $U(2)$ [W], thereby showing that symplectic analogues of spherical varieties need not be Kaehler. Similar results have been independently obtained by F. Knop. However it appears that Tolman's manifold does admit a non-invariant Kaehler structure [To2].

The example below is a twelve dimensional compact symplectic manifold with a Hamiltonian circle action which has one isolated fixed point and two connected fixed submanifolds of dimensions four and ten respectively. For this manifold $M$, the third Betti number is odd. Thus $M$ admits no Kaehler structure, invariant or otherwise.

\section{The construction}

McDuff $[\mathrm{McD}]$ showed that if one embeds symplectically a certain 4 manifold $N$ into the standard projective 5 -space $\mathbb{C} P^{5}$ and then blows up $\mathbb{C} P^{5}$ symplectically along $N$, the resulting manifold $\widetilde{\mathbb{C P}}_{N}^{5}$ has an odd third Betti number and hence admits no Kaehler structure $(N$ is the KodairaThurston example of a symplectic non-Kaehler manifold [K, Theorem 19], [Th]). Consider the action of the circle $S^{1}$ on $\mathbb{C} P^{6}$ given by

$$
\lambda \cdot\left[z_{0}, z_{1}, \ldots, z_{6}\right]=\left[\lambda z_{0}, z_{1}, \ldots, z_{6}\right] .
$$

The action is Hamiltonian, and is free away from the point $p=[1,0, \ldots 0]$ and the hyperplane $H=\left\{\left[0, z_{1}, \ldots, z_{6}\right]\right\} \simeq \mathbb{C} P^{5}$. Embed the manifold $N$ (symplectically and equivariantly) into $\mathbb{C} P^{6}$ by embedding it into the hyperplane $H$.

Guillemin and Sternberg pointed out that symplectic blowing up of submanifolds can be done equivariantly [GS]. Blow up $\mathbb{C} P^{6}$ along $N$ equivariantly and symplectically. The result is a compact symplectic manifold $M$ with a Hamiltonian circle action. It is not hard to see that the fixed point set $M^{S^{1}}$ has three components: the point $p$, the manifold $\widetilde{\mathbb{C P}}_{N}^{5}$ and a copy of $N$. Since the manifold $N$ admits no Kaehler structure, it follows immediately that $M$ admits no invariant Kaehler structure.

To show that $M$ admits no Kaehler structure whatsoever, we use Morse theory. The moment map of the action of $S^{1}$ on $M$ is Bott-Morse and its critical set is precisely the set of fixed points $M^{S^{1}}$. It follows from an elementary argument (relative Morse lemma, Mayer-Vietoris and excision) 
that $H^{3}(M, \mathbb{R}) \simeq H^{3}\left(\widetilde{\mathbb{C P}}_{N}^{5}, \mathbb{R}\right)=\mathbb{R}^{3}$.

\section{Conclusions}

By a theorem of Gromov and Tischler [Gr], [Ti], any compact integral symplectic manifold $X$ of dimension $n$ can be symplectically embedded in $\mathbb{C} P^{n+1}$. The above construction produces a compact symplectic manifold $M_{X}$ with a Hamiltonian $S^{1}$ action that has $X$ as one of the components of the fixed point set. Thus if the manifold $X$ admits no complex structure [FGG], [Ge] then the manifold $M_{X}$ admits no invariant complex structure; if the manifold $X$ admits no polarization [Got] then the manifold $M_{X}$ admits no invariant polarization; and so on.

\section{Acknowledgements}

The idea for the construction of a symplectic manifold with a Hamiltonian circle action and no Kaehler structure came to me in a conversation with Yael Karshon. Sue Tolman helped me with the cohomology computations.

A large part of the work on this project was done at MIT. The author is grateful to MIT and the NSF for their support.

\section{References}

[D] T. Delzant, Hamiltoniens périodiques et images convexes de l'application moment, Bull. Math. France 116 (1988), 315-339.

[FGG] M. Fernándes, M. J. Gotay, and A. Gray, Compact parallelizable fourdimensional symplectic and complex manifolds, Proc. Amer. Math. Soc. 103 (1988), 1209-1212.

[Ge] H. Geiges, Symplectic structures on $T^{2}$ bundles over $T^{2}$, Duke Math. J. 67 (1992), 539-555.

[Gom] R. E. Gompf, A new construction of symplectic manifolds, Ann. of Math. 142 (1995), 527-598.

[Got] M. J. Gotay, A class of non-polarizable symplectic manifolds, Monatsch. Math. 103 (1987), 27-30.

[Gr] M. Gromov, Partial differential relations, Springer Verlag; New York, Berlin, 1986.

[GS] V. Guillemin and S. Sternberg, Birational equivalence in the symplectic category, Invent. Math. 97 (1989), 485-522.

[Ka] Y. Karshon, Periodic Hamiltonian flows on four dimensional manifolds, dg-ga/9510004, URL http://xxx.lanl.gov/abs/dg-ga/9510004.

[K] K. Kodaira, On the structure of compact complex analytic surfaces I, Amer. J. Math. 86 (1964), 751-798.

[McD] D. McDuff, Examples of simply connected non Kaehlerian manifolds, J. Diff. Geom. 20 (1984), 267-277.

[Ti] D. Tischler, Closed 2-forms and an embedding theorem for symplectic manifolds, J. Diff. Geom. 12 (1977), 229-235. 
[Th] W. P. Thurston, Some simple examples of symplectic manifolds, Proc. Amer. Math. Soc. 55 (1976), 467-468.

[To] S. Tolman, Examples of non-Kaehler Hamiltonian torus actions, dg-ga/9511007, URL http://xxx.lanl.gov/abs/dg-ga/9511007.

[To2] ㄴ. personal communication, November 1995.

[W] C. Woodward, Multiplicity-free Hamiltonian actions need not be Kähler, dg-ga/9506009, URL http://xxx.lanl.gov/abs/dg-ga/9506009.

Department of Mathematics, University of Illinois, Champaign, IL 61801

E-mail address: lerman@math.uiuc.edu 